\title{
Botany
}

American Journal of Botany: e470-e473. 2012.

AJB Primer Notes \& Protocols in the Plant Sciences

\section{A SET OF PLASTID MICROSATELLITE LOCI FOR THE GENUS DYCKIA (BROMELIACEAE) DERIVED FROM 454 PYROSEQUENCING ${ }^{1}$}

\author{
Florian Krapp ${ }^{2}$, Tina Wöhrmann ${ }^{2}$, Diego Sotero de Barros Pinangé ${ }^{3}$, Ana Maria \\ BenKo-Iseppon ${ }^{3}$, BRuno HuetTel $^{4}$, AND Kurt Weising ${ }^{2,5}$
}

\begin{abstract}
${ }^{2}$ Plant Molecular Systematics, Department of Sciences, University of Kassel, D-34132 Kassel, Germany; ${ }^{3}$ Genetics Department, CCB, Universidade Federal de Pernambuco (UFPE), Av. Prof. Moraes Rego 1235, 50670-420, Recife, Pernambuco, Brazil; and

${ }^{4}$ Max Planck Institute for Plant Breeding Research, Carl-von-Linné-Weg 10, 50829 Cologne, Germany

- Premise of the study: Phylogeographical analyses of Dyckia (Bromeliaceae) suffer from low levels of sequence variation. Plastid microsatellite markers were developed to achieve a better-resolved genus-wide plastid genealogy of Dyckia.

- Methods and Results: Approximately $84 \%$ of the D. marnier-lapostollei plastome was sequenced using 454 technology. Flanking primer pairs were designed for 34 out of 36 chloroplast simple sequence repeats (cpSSRs) detected, and 12 loci were further characterized by genotyping Dyckia samples at the level of populations and species. Three, five, and six cpSSRs were polymorphic among four individuals of $D$. limae, 12 individuals of $D$. dissitiflora, and 12 of $D$. pernambucana, respectively, with two to three alleles per locus and species. All loci were polymorphic among 19 different Dyckia species, with three to 10 alleles per locus. Ten primer pairs cross-amplified with bromeliad genera from five subfamilies.

- Conclusions: The set of 12 cpSSR markers provides a toolbox to analyze phylogeographical patterns of Dyckia species.
\end{abstract}

Key words: Bromeliaceae; cpSSR; Dyckia; plastome; population genetics.

The genus Dyckia Schult. f. (Bromeliaceae) currently comprises 147 described species of xerophytic, terrestrial, or epilithic rosette plants with showy yellow, red, or orange flowers (Smith and Downs, 1974). The genus is distributed across eastern South America, with a center of diversity in the cerrado biome of Brazil and adjacent countries. Species of Dyckia and of its closest relative Encholirium Mart. ex Schult. f. typically inhabit azonal, arid, or rupicolous habitats that are characterized by poor soil, little water supply, high temperatures, and strong sun exposure. Pollination is mainly by hummingbirds and insects. Fruits are capsules that release winged, wind-dispersed seeds upon maturity (Smith and Downs, 1974).

Little is known about infrageneric relationships within $D y$ ckia, the genetic structure and variation within its species, and the mechanisms of speciation. This paucity of information is in part due to the fact that many Dyckia species are rare and narrow endemics, which are barely represented in herbaria and living collections. Some species are even known from their type locality only. Another problem is the high degree of intraspecific morphological plasticity, which makes species

${ }^{1}$ Manuscript received 23 March 2012; revision accepted 19 June 2012.

The authors thank J. Peters, N. Schütz, and the Botanical Gardens of Heidelberg, Bonn, and Vienna for providing plant material, as well as R. B. Louzada, G. Cruz, and A. M. Wanderley for help during field work. F.K. and D.S.B.P. are supported by fellowship grants of the Otto-Braun-Fonds (Melsungen) and the Fundação de Amparo à Ciência e Tecnologia do Estado de Pernambuco (FACEPE), respectively. This work was supported by PNADB/CAPES and DAAD/CAPES in the frame of a PROBRAL project.

${ }^{5}$ Author for correspondence: weising@ uni-kassel.de

doi:10.3732/ajb.1200153 delimitation in Dyckia notoriously difficult. We have initiated a genus-wide molecular phylogenetic study of Dyckia, based on plastid and nuclear DNA sequences. Our preliminary results indicate very low levels of plastid sequence divergence, suggesting a young age of the genus (Krapp, unpublished data). Whereas chloroplast haplotypes are often shared between species, haplotype networks based on plastid DNA show a pronounced geographical pattern across the distributional range of the genus. Chloroplast simple sequence repeats (cpSSRs), also called chloroplast microsatellites, are among the most sensitive tools for evaluating plastid DNA variation (Ebert and Peakall, 2009). To achieve a better-resolved genus-wide plastid phylogeography of Dyckia, we developed a set of 12 polymorphic cpSSR markers based on de novo 454 sequencing.

\section{METHODS AND RESULTS}

Total genomic DNA was isolated from one individual plant of Dyckia marnier-lapostollei L. B. Sm. var. estevesii Rauh from Goiania, central Brazil (see Appendix 1), using a modified cetyltrimethylammonium bromide (CTAB) procedure (Tel-Zur et al., 1999). This species was chosen because its plastid haplotype takes a central position in a statistical parsimony network, suggesting an ancestral state within the genus (Krapp, unpublished results). Fragmentation of a 5- $\mu \mathrm{g}$ DNA aliquot by nebulization, preparation of barcoded libraries, and shotgun sequencing on a Roche 454 GS-FLX with the Titanium Sequencing Kit XLR70 and the Titanium PicoTiterPlate Kit (Roche Diagnostics, Penzberg, Germany) were performed as described previously (Wöhrmann et al., 2012a). Altogether, 59624 reads were obtained from three independent runs. The proportion of a single 454 sequencing lane devoted to D. marnier-lapostollei was $4.2 \%, 2.1 \%$, and $4.1 \%$ in the first, second, and third run, respectively. Sequences of plastid origin were identified using the BLAST function of the software package Geneious (Drummond et al., 2010). The fully sequenced plastome of Typha latifolia L. (Guisinger et al., 2010) 
TABLE 1. Characteristics of 12 chloroplast microsatellite primer pairs developed in Dyckia marnier-lapostollei var. estevesii.

\begin{tabular}{|c|c|c|c|c|c|}
\hline Locus & Primer sequences $\left(5^{\prime}-3^{\prime}\right)$ & Position & Repeat motif & Size (bp) & GenBank accession no. ${ }^{a}$ \\
\hline DSSR-L01 & $\begin{array}{l}\mathrm{F}: \text { GTCAATTTTCAAGTTCAGCC } \\
\mathrm{R}: \text { TCACGATTTCATCTACTTGC }\end{array}$ & $\begin{array}{l}a t p \mathrm{~B}-r b c \mathrm{~L} \\
\text { intergenic }\end{array}$ & $(\mathrm{T})_{13} \mathrm{C}(\mathrm{A})_{10}$ & 75 & JQ743912 \\
\hline DSSR-L04 & $\begin{array}{l}\mathrm{F}: \text { AAAGGATGAGATCAATTCGG } \\
\mathrm{R}: \text { AAGATACATCGGAAAGTCCC }\end{array}$ & $\begin{array}{l}n d h \mathrm{~A} \\
\text { intronic }\end{array}$ & $(\mathrm{T})_{9} *$ & 94 & JQ743913 \\
\hline DSSR-L06 & $\begin{array}{l}\mathrm{F}: \text { ATtGATTGAATAAACCGGGG } \\
\mathrm{R}: \text { TAAATAAGAAATTGGAATGG }\end{array}$ & $\begin{array}{l}\text { trnK-UUU-rps } 16 \\
\text { intergenic }\end{array}$ & $(\mathrm{T})_{13}$ & 77 & JQ743914 \\
\hline DSSR-N01 & $\begin{array}{l}\mathrm{F}: \text { GTTCCCAGTAAGAACCAACC } \\
\mathrm{R}: \text { CTCAATAATTTCACATTTCC }\end{array}$ & $\begin{array}{c}\text { rpo } \mathrm{C} 1 \\
\text { intronic }\end{array}$ & $(\mathrm{T})_{14}$ & 102 & JQ743915 \\
\hline DSSR-N04 & $\begin{array}{l}\mathrm{F}: \text { GAAATCAATGTGTCGATTCC } \\
\mathrm{R}: \text { TTTNAATAGAAAGAAGACCC }\end{array}$ & $\begin{array}{l}c l p \mathrm{P} \\
\text { intronic }\end{array}$ & $(\mathrm{T})_{11}$ & 87 & JQ743916 \\
\hline DSSR-N05 & $\begin{array}{l}\mathrm{F}: \text { TGAGATGAGTTTTGGCTCCC } \\
\mathrm{R}: \text { AACAATACATCAATGATAGG }\end{array}$ & $\begin{array}{c}c l p \mathrm{P} \\
\text { intronic }\end{array}$ & $(\mathrm{A})_{12}$ & 85 & JQ743917 \\
\hline DSSR-N07 & $\begin{array}{l}\mathrm{F}: \text { ATTATATACATCTGAAACCC } \\
\mathrm{R}: \text { CTTCCTCCTGAGCATTACGG }\end{array}$ & $\begin{array}{l}\text { trnP-UGG- } p s a \mathrm{~J} \\
\text { intergenic }\end{array}$ & $(\mathrm{A})_{13}$ & 74 & JQ743918 \\
\hline DSSR-N10 & $\begin{array}{l}\mathrm{F}: \text { TNAATCAATATGGCGAAGGC } \\
\mathrm{R}: \text { ATTCCCTCACGCTTGGCGCC }\end{array}$ & $\begin{array}{c}\operatorname{clp} \mathrm{P} \\
\text { intronic }\end{array}$ & $(\mathrm{T})_{10}$ & 79 & JQ743919 \\
\hline DSSR-N11 & $\begin{array}{l}\mathrm{F}: \text { ATAGATAAAATTATCGGGCC } \\
\mathrm{R}: \text { AAATTTAAACTACATATTCC }\end{array}$ & $\begin{array}{l}n d h \mathrm{G}-n d h \mathrm{I} \\
\text { intergenic }\end{array}$ & $(\mathrm{A})_{18}$ & 100 & JQ743920 \\
\hline DSSR-N15 & $\begin{array}{l}\mathrm{F}: \text { CTTCCATTTAтCCATAтCCC } \\
\mathrm{R}: \text { AAAATAAATCTGATTATGGG }\end{array}$ & $\begin{array}{l}r p l 16 \\
\text { intronic }\end{array}$ & $(\mathrm{T})_{11}$ & 64 & JQ743921 \\
\hline DSSR-N16 & $\begin{array}{l}\mathrm{F}: \text { TTATACCAAATGATCAATCG } \\
\mathrm{R}: \text { ACTCTTTCATTCTTTTCCG }\end{array}$ & $\begin{array}{l}\text { rpl16-rps3 } \\
\text { intergenic }\end{array}$ & $(\mathrm{T})_{13}$ & 90 & JQ743922 \\
\hline DSSR-N18 & $\begin{array}{l}\mathrm{F}: \text { AAATAGGTAATCTATTCCCC } \\
\mathrm{R}: \text { GTAAGCATTACACAATCTCC }\end{array}$ & $\begin{array}{l}p s b \mathrm{~K}-p s b \mathrm{I} \\
\text { intergenic }\end{array}$ & $(\mathrm{A})_{15}$ & 63 & JQ743923 \\
\hline
\end{tabular}

${ }^{\mathrm{a}}$ GenBank accession numbers of the sequences on which the primers are based.

* The SSR motif at DSSR-L04 had only nine T residues in D. marnier-lapostollei, but had up to 14 T residues in other Dyckia species for which sequence data were available for primer design.

was taken as a reference. A total of 3826 plastid reads were assembled into 77 contigs and 12 singletons, which together represent 113449 bases of the $D$. marnier-lapostollei plastome (counting the two inverted repeats only once). This corresponds to an overall coverage of $\sim 84 \%$, when compared to the T. latifolia plastome. A total of 36 mononucleotide repeats with $\geq 10$ bases were detected (181 with $\geq 7$ bases) using the FIND function of PhyDE (Müller et al., 2010). Besides two short dinucleotide repeats, each with an (AT) motif, no other types of SSRs were observed. Flanking primer pairs were designed by eye for 34 loci, with a default length of 20 nucleotides and a GC clamp of up to three nucleotides at the $3^{\prime}$ end. For three loci (DSSR-L01, DSSR-L04, and DSSR-L06; Table 1), consensus primers were derived from alignments of the D. marnier-lapostollei sequence with sequence data previously generated by Sanger sequencing of the same loci in other Dyckia species (Krapp, unpublished data).

Primer functionality was initially tested on a single accession each of D. marnier-lapostollei, D. dissitiflora Schult. f., and D. pernambucana L. B. Sm. PCRs were carried out in 10- $\mu \mathrm{L}$ volumes using a Biometra T1-cycler or a Biometra T-Gradient cycler (Biometra GmbH, Göttingen, Germany), using

TABLE 2. Observed allele sizes at 12 chloroplast microsatellite loci in three populations of $D$. dissitiflora and D. pernambucana and one population of D. limae, allele numbers and size range in 19 different Dyckia species (one individual each), and cross-amplification in eight additional genera of Bromeliaceae (see Appendix 1).

\begin{tabular}{|c|c|c|c|c|c|c|c|c|c|c|c|c|c|c|c|c|}
\hline \multirow[b]{3}{*}{ Locus } & \multicolumn{7}{|c|}{ Allele sizes } & & & \multirow{2}{*}{\multicolumn{7}{|c|}{$\begin{array}{l}\text { Cross-amplification in other bromeliad } \\
\text { genera }^{\dagger}\end{array}$}} \\
\hline & \multicolumn{3}{|c|}{ D. dissitiflora } & \multicolumn{3}{|c|}{ D. pernambucana } & \multirow{2}{*}{$\begin{array}{c}\text { D. limae } \\
\text { Jerusalém* } \\
(N=4)\end{array}$} & \multicolumn{2}{|c|}{19 Dyckia species } & & & & & & & \\
\hline & $\begin{array}{l}\text { Cachoeira* } \\
(N=4)\end{array}$ & $\begin{array}{l}\text { Lajes* } \\
(N=4)\end{array}$ & $\begin{array}{l}\text { Morrão* } \\
(N=4)\end{array}$ & $\begin{array}{l}\text { Aldeia* } \\
(N=4)\end{array}$ & $\begin{array}{l}\text { Brejo* } \\
(N=4)\end{array}$ & $\begin{array}{l}\text { Papagaio* } \\
(N=4)\end{array}$ & & No. of alleles & $\begin{array}{l}\text { Size range } \\
\text { (bp) }\end{array}$ & En & De & Fo & $\mathrm{Pi}$ & $\mathrm{Pu}$ & An & $\mathrm{He} \mathrm{Ti}$ \\
\hline DSSR-L01 & 75 & 75,76 & 75,76 & 77 & 75,76 & 77 & 76,77 & 9 & $72-80$ & + & + & + & + & + & + & + \\
\hline DSSR-L04 & 98 & 98 & 98 & 99 & 97 & 98 & 99 & 6 & $94-99$ & + & + & + & + & + & + & ++ \\
\hline DSSR-N05 & 86 & 86 & 86 & 85,86 & 85,86 & 86 & 85,86 & 4 & $84-87$ & + & + & + & + & + & + & ++ \\
\hline DSSR-N07 & 74 & 74 & 74 & 74 & 74 & 74 & 74 & 5 & $71-75$ & + & + & - & + & + & + & ++ \\
\hline DSSR-N10 & 81 & 79 & 79,81 & 79 & 79 & 79 & 79 & 3 & $79-81$ & + & + & + & + & + & + & ++ \\
\hline DSSR-N11 & 99 & 96100 & 99 & 97 & 98 & 98 & 98 & 10 & 94-104 & + & + & + & - & - & - & + \\
\hline DSSR-N15 & 65 & 65 & 65 & 65 & 65 & 65 & 65 & 3 & $64-66$ & + & + & + & + & + & + & ++ \\
\hline DSSR-N16 & 90 & 90 & 90 & 90 & 90 & 90 & 90 & 5 & $87-91$ & + & + & + & + & + & + & ++ \\
\hline
\end{tabular}

Note $+=$ amplification; - = no amplification; An = Ananas (Bromelioideae); De = Deuterocohnia $;$ En = Encholirium; Fo = Fosterella $; \mathrm{Pi}=$ Pitcairnia (all Pitcairnioideae); $\mathrm{He}=$ Hechtia (Hechtioideae); $\mathrm{Pu}=$ Puya (Puyoideae); Ti = Tillandsia (Tillandsoideae).

* Locality information for the populations is provided in Appendix 1.

${ }^{\dagger}$ Single PCR product in the expected size range. 
the indirect fluorescence labeling procedure described by Schuelke (2000). Each assay contained approximately $1 \mathrm{ng}$ of template DNA, $1 \times$ Mango-Taq reaction buffer (Bioline, Taunton, Massachusetts, USA), $1.5 \mathrm{mM} \mathrm{MgCl}_{2}, 0.2 \mathrm{mM}$ of each dNTP, $0.04 \mu \mathrm{M}$ forward primer carrying a 5'-M13 tail, $0.16 \mu \mathrm{M}$ of M13 forward primer with fluorescent $5^{\prime}$-IRD700 modification, $0.16 \mu \mathrm{M}$ unlabeled reverse primer, $0.5 \mu \mathrm{g} / \mu \mathrm{L}$ BSA, and $0.05 \mathrm{U}$ Mango-Taq DNA polymerase (Bioline). All loci were amplified using a standard PCR program with an initial denaturation at $80^{\circ} \mathrm{C}$ for $5 \mathrm{~min}$, followed by 30 cycles of denaturation at $94^{\circ} \mathrm{C}$ for 1 min, primer annealing at $52^{\circ} \mathrm{C}$ for $1 \mathrm{~min}$, and elongation at $65^{\circ} \mathrm{C}$ for $2 \mathrm{~min}$. Final extension was performed at $65^{\circ} \mathrm{C}$ for $10 \mathrm{~min}$. Samples were electrophoresed on denaturing $6 \%$ polyacrylamide gels in $1 \times$ TBE buffer, using an automated sequencer (Li-Cor 4200 IR $^{2}$; Li-Cor Biosciences, Bad Homburg, Germany). Fragment sizes were determined by visual examination with the help an external size standard, as outlined by Wöhrmann et al. (2012a). Allele sizes were validated by repeated PCRs of subsets of samples using either Mango-Taq polymerase or a set of proofreading polymerases (Long High Fidelity Enzyme Mix; Rovalab, Teltow, Germany), following the protocol supplied by the manufacturer.

The 12 most polymorphic cpSSR loci were used to genotype (1) population samples from D. limae L. B. Sm., D. dissitiflora, and D. pernambucana; (2) single accessions from 16 additional Dyckia species; and (3) one or two species each of eight bromeliad genera belonging to five subfamilies. Dyckia dissitiflora was chosen as an example of a Dyckia species with a relatively large distribution range across Brazil, whereas $D$. limae and $D$. pernambucana were taken as a typical example of two species that are not clearly distinguishable by morphological characters. Locus characteristics, primer sequences, and GenBank accession numbers of these 12 markers are summarized in Table 1, fragment sizes for all samples and loci are compiled in Table 2, and all plant materials used in this study are listed in Appendix 1.

Three, five, and six cpSSR loci were polymorphic among four individuals of D. limae, 12 individuals from three populations of $D$. dissitiflora, and 12 individuals from three populations of $D$. pernambucana, respectively (Table 2). Two to three alleles were observed per locus and species. All loci were highly polymorphic at the species level, with three to 10 alleles per locus across 19 Dyckia species (Table 2). Allele size distributions were generally compatible, with a variable number of mononucleotide repeats being the molecular basis for size variation. Overall, only six out of 540 individual PCRs performed with any Dyckia species failed. All loci produced single PCR fragments within the expected size range in the closely related genera Encholirium and Deuterocohnia $\mathrm{Mez}$, and nine of the 12 primer pairs successfully cross-amplified in six other genera from five subfamilies of Bromeliaceae (Table 2).

\section{CONCLUSIONS}

The set of 12 novel cpSSR markers presented here provides a promising toolbox for reconstructing plastid genealogies and elucidating phylogeographical patterns within Dyckia. In conjunction with nuclear SSR markers that are currently being developed in our group (Wöhrmann et al., 2012b), the cpSSRs are also promising candidates for population genetic analyses in $D$. dissitiflora, D. limae, $D$. pernambucana, and probably many other Dyckia species. Primer binding sites appear to be well-conserved among Bromeliaceae, suggesting that the $12 \mathrm{cpSSR}$ markers may be applicable for genetic studies throughout the family.

\section{LITERATURE CITED}

Drummond, A. J., B. Ashton, M. Cheung, J. Heled, M. Kearse, R. Moir, S. Stones-Havas, et al. 2010. Geneious v5.0. Website http://www. geneious.com [accessed 3 December 2010].

Ebert, D., and R. Peakall. 2009. Chloroplast simple sequence repeats (cpSSRs): Technical resources and recommendations for expanding cpSSR discovery and applications to a wide array of plant species. Molecular Ecology 9: 673-690.

Guisinger, M. M., T. W. Chumley, J. V. Kuehl, J. L. Boore, and R. K. JANSEN. 2010. Implications of the plastid genome sequence of Typha (Typhaceae, Poales) for understanding genome evolution in Poaceae. Journal of Molecular Evolution 70: 149-166.

Müller, J., K. Müller, and D. Quandt. 2010. PhyDE: Phylogenetic data editor. Version 0.9971. Program distributed by the author. Website http://www.phyde.de/ [accessed 23 November 2010].

SchuelKe, M. 2000. An economic method for the fluorescent labeling of PCR fragments. Nature Biotechnology 18: 233-234.

Smith, L. B., AND R. J. Downs. 1974. Pitcairnioideae (Bromeliaceae). Flora Neotropica Monographs 14, part 1. New York Botanical Garden, Bronx, New York, USA.

Tel-Zur, N., S. Aвbo, D. Myslabodski, and Y. Mizrahi. 1999. Modified CTAB procedure for DNA isolation from epiphytic cacti of the genera Hylocereus and Selenicereus (Cactaceae). Plant Molecular Biology Reporter 17: 249-254.

Wöhrmann, T., N. Wagner, F. Krapp, B. Huettel, and K. Weising, 2012a. Development of microsatellite markers in Fosterella rusbyi (Bromeliaceae) using 454 pyrosequencing. American Journal of Botany 99: e160-e163.

Wöhrmann, T., D. S. B. Pinangé, F. Krapp, A. M. Benko-Iseppon, B. Huettel, And K. Weising. 2012b. Development of 15 nuclear microsatellite markers in the genus Dyckia (Pitcairnioideae; Bromeliaceae) using 454 pyrosequencing. Conservation Genetics Resources DOI: 10.1007/s12686-012-9738-y. 
APPENDIX 1. Plant material used for this study.

\begin{tabular}{|c|c|c|c|}
\hline Species & Collector (Herbarium) ${ }^{\mathrm{a}}$ & Location $^{\mathrm{b}}$ & GPS coordinates \\
\hline $\begin{array}{l}\text { Dyckia dissitiflora Schult. f. } \\
\text { Pop. Cachoeira }(N=4)\end{array}$ & A. M. Iseppon, Pinangé, D. \& Cruz, G. 1605 (UFP) & Cachoeira “Ferro Doido," Bahia (BR) & $-11.6279 ;-41.0005$ \\
\hline $\begin{array}{l}\text { Dyckia dissitiflora Schult. f. } \\
\text { Pop. Lajes }(N=4)\end{array}$ & A. M. Iseppon, Pinangé, D. \& Cruz, G. 1598 (UFP) & Lajes, Bahia (BR) & $-11.6010 ;-41.1645$ \\
\hline $\begin{array}{l}\text { Dyckia dissitiflora Schult. f. } \\
\text { Pop. Morrão }(N=4)\end{array}$ & A. M. Iseppon, Pinangé, D. \& Cruz, G. 1562 (UFP) & Morrão, Bahia (BR) & $-11.5901 ;-41.2072$ \\
\hline $\begin{array}{l}\text { Dyckia limae L. B. Sm. } \\
\text { Pop. Jerusalém }(N=4)\end{array}$ & A. M. Wanderley s.n. (UFP) & Serra de Jerusalém, Pernambuco (BR) & $-8.5837 ;-37.2384$ \\
\hline $\begin{array}{l}\text { Dyckia pernambucana L. B. Sm. } \\
\text { Pop. Aldeia }(N=4)\end{array}$ & D. Pinangé et al. DCKB/09.2009 (UFP) & Aldeia Couro d'Anta, Pernambuco (BR) & $-8.3254 ;-36.7562$ \\
\hline $\begin{array}{l}\text { Dyckia pernambucana L. B. Sm. } \\
\text { Pop. Brejo }(N=4)\end{array}$ & D. Pinangé et al. DKCA/09.2009 (UFP) & $\begin{array}{l}\text { Brejo da Madre de Deus, Pernambuco } \\
\text { (BR) }\end{array}$ & $-8.1894 ;-36.3931$ \\
\hline $\begin{array}{l}\text { Dyckia pernambucana L. B. Sm. } \\
\text { Pop. Papagaio }(N=4)\end{array}$ & A. M. Wanderley s.n. (UFP) & Pico do Papagaio, Pernambuco (BR) & $-7.8228 ;-38.0554$ \\
\hline Dyckia aff. brevifolia Baker & P. Braun 840 (HD) & Itacambira, Minas Gerais (BR) & $-17.0667 ;-43.3000$ \\
\hline Dyckia estevesii Rauh & P. Braun s.n. (HD) & $\mathrm{BR}$ & NA \\
\hline Dyckia ferox Mez & W. Rauh 64237 (HD) & Cerro Colorado, Cordoba (RA) & $-30.1000 ;-63.9333$ \\
\hline Dyckia goehringii E. Gross \& Rauh & W. Rauh 67622 (HD) & Diamantina, Minas Gerais (BR) & $-18.2500 ;-43.6000$ \\
\hline Dyckia granmogulensis Rauh & W. Rauh 56484 (HD) & Grão Mogol, Minas Gerais (BR) & $-16.5667 ;-42.9000$ \\
\hline Dyckia aff. ibiramensis Reitz & L. Horst 1287 (HD) & Diamantina, Minas Gerais (BR) & $-18.2500 ;-43.6000$ \\
\hline Dyckia leptostachya Baker & H. Amerhauser s.n. (WU) & Caacupé, Cordillera (PY) & $-25.3833 ;-57.1500$ \\
\hline Dyckia lindevaldae Rauh & P. Braun BR 691 (HD) & Alto Paraiso, Goiás (BR) & $-14.1167 ;-47.5167$ \\
\hline Dyckia macedoi L. B. Sm. & R. B. Louzada, Pinangé, D. \& Medeiros, M. 151 (SP) & Santana do Riacho, Minas Gerais (BR) & $-19.3539 ;-43.6237$ \\
\hline $\begin{array}{l}\text { Dyckia marnier-lapostollei var. } \\
\text { estevesii Rauh }\end{array}$ & L. Horst 5 (HD) & Goiania, Goiás (BR) & $-16.6667 ;-49.2667$ \\
\hline Dyckia marnier-lapostollei L. B. Sm. & L. Horst 4 (HD) & Cristalina, Goiás (BR) & $-16.7500 ;-47.6000$ \\
\hline Dyckia microcalyx Baker & W. Till 6020 (WU) & Cerros Acahay, Paraguari (PY) & $-25.9167 ;-57.1500$ \\
\hline Dyckia aff. pumila L. B. Sm. & P. Braun BR 696 (HD) & Ponte Branca, Mato Grosso (BR) & $-16.4500 ;-52.6667$ \\
\hline $\begin{array}{l}\text { Dyckia remotiflora var. indet. } \\
\text { Otto \& A. Dietr. }\end{array}$ & L. Horst s.n. (HD) & $\mathrm{BR}$ & NA \\
\hline Dyckia tobatiensis Hassl. & W. \& S. Till 6050 (WU) & Tobati, Cordillera (PY) & $-25.2500 ;-57.0667$ \\
\hline Dyckia velascana Mez & W. \& S. Till 5012 (WU) & Ascochinga, Cordoba (RA) & $-30.9500 ;-64.2667$ \\
\hline Dyckia vestita Hassl. & W. \& S. Till 6018 (WU) & Paraguari, Paraguari (PY) & $-25.6333 ;-57.1500$ \\
\hline Encholirium horridum L. B. Sm. & W. Schindhelm s.n. (HD) & Pedra Azul, Minas Gerais (BR) & $-15.9867 ;-41.4069$ \\
\hline Encholirium magalhaesii L. B. Sm. & s.n. (BONN) & $\mathrm{BR}$ & NA \\
\hline $\begin{array}{l}\text { Deuterocohnia brevispicata } \\
\text { Rauh \& L. Hrom. }\end{array}$ & N. Schütz 06/028 (FR) & Florida, Santa Cruz (BOL) & $-18.0154 ;-64.1001$ \\
\hline Deuterocohnia glandulosa E. Gross & N. Schütz 06/019 (FR) & Ipati, Santa Cruz (BOL) & $-19.7063 ;-63.6521$ \\
\hline Fosterella villosula (Harms) L. B. Sm. & J. Peters 06.0105 (HD) & Cochabamba, Cochabamba (BOL) & $-17.0611 ;-65.6444$ \\
\hline $\begin{array}{l}\text { Fosterella weddelliana (Brongn. ex Baker) } \\
\text { L. B. Sm. }\end{array}$ & M. Miyagawa s.n. (HD) & Solacana (BOL) & NA \\
\hline $\begin{array}{l}\text { Pitcairnia feliciana (A. Chev.) Harms \& } \\
\text { Mildbr. }\end{array}$ & I. Ebert \& D. Bangoura s.n. ex coll. P. Bak (WU) & RG & NA \\
\hline Pitcairnia heterophylla (Lindl.) Beer & K. Senghas O-11230 (HD) & Cruz de Ocotte, Guerrero (MEX) & $17.5500 ; 99.8833$ \\
\hline Puya ferruginea (Ruiz \& Pav.) L. B. Sm. & W. Rauh s.n. (HD) & Rio Marañon (PE) & NA \\
\hline Puya herzogii Wittm. & T. Krömer 6581 (HD) & Carrasco, Cochabamba (BOL) & $-17.1933 ;-64.9731$ \\
\hline Ananas ananassoides (Baker) L. B. Sm. & P. Maas s.n. (HD) & Est. Amazonas (BR) & NA \\
\hline Hechtia caerulea (Matuda) L. B. Sm. & W. Rauh s.n. (HD) & Est. Mexico (MEX) & NA \\
\hline Tillandsia usneoides (L.) L. & W. Rauh s.n. (HD) & Yungas Cachi (RA) & NA \\
\hline
\end{tabular}

Note: $N=$ population size; $\mathrm{NA}=$ not available.

${ }^{a}$ Herbaria: BONN = University of Bonn; FR = Senckenberg Research Institute, Frankfurt; HD = Botanical Garden of Heidelberg; SP = Instituto de Botânica; São Paulo; UFP = Universidade Federal de Pernambuco; WU = University of Vienna.

${ }^{\mathrm{b}} \mathrm{BOL}=$ Bolivia $; \mathrm{BR}=$ Brazil MEX = Mexico; $\mathrm{PE}=$ Peru; $\mathrm{PY}=$ Paraguay; $\mathrm{RA}=$ Argentina $; \mathrm{RG}=$ Guinea. 